\title{
Associação entre a necessidade de prescrição de correção óptica e outras doenças oculares em crianças na idade escolar
}

\author{
Association between the need for optical correction prescription and \\ other ocular conditions in school children
}

\author{
Maria Isabel Lynch Gaete ${ }^{1}$ \\ Rodrigo Pessoa Cavalcanti Lira ${ }^{2}$ \\ Luiz Felipe Lynch de Moraes ${ }^{3}$ \\ Mirelle Souza Leão Vasconcelos ${ }^{4}$ \\ Cristiano Viana de Oliveira ${ }^{5}$
}

\begin{tabular}{l} 
RESUMO \\
\hline Objetivos: Verificar a relação entre a prescrição de óculos e a presença de \\
afecções oculares encontradas em crianças na idade escolar. Métodos: \\
Crianças na idade escolar que foram examinadas na Campanha “Olho no \\
Olho” em Recife no ano de 2004 . Foram seguidas etapas recomendadas \\
pelo Conselho Brasileiro de Oftalmologia para realização desta Cam- \\
panha, desde a preparação dos professores para triagem das crianças até \\
a consulta com os oftalmologistas. Os pacientes foram divididos em dois \\
grupos de acordo com a necessidade de prescrição de óculos, sendo \\
relacionados com a presença de doença ocular ou não. Trata-se de um \\
estudo descritivo de delineamento transversal que teve seus resultados \\
analisados a partir do programa estatístico Epi Info versão 6.0 . Resulta- \\
dos: De uma amostra de 1.994 escolares, 686 deles apresentaram necessi- \\
dade de óculos (34,4\% Grupo1), sendo que 543 (27,2\%) não apresenta- \\
ram qualquer doença ocular, enquanto 143 (7,17\%) tinham alguma doen- \\
ça ocular. Em 1.308 crianças (65,5\% Grupo 2 ) não houve necessidade de \\
óculos. Destas, 1.256 (62,5\%) não apresentavam doença oftalmológica, \\
enquanto 52 (2,6\%) apresentavam algum tipo de afecção ocular. Os \\
grupos 1 e 2 foram comparados entre si verificando que crianças que \\
necessitam de óculos apresentam um risco relativo de possuírem doença \\
ocular de 5,24 (Intervalo de Confiança de 95\%: 3,87 a 7,10 ) vezes maior \\
que as crianças que não precisam dos mesmos, com diferença estatistica- \\
mente significativa entre os dois grupos (p= 0.0000001 ). Conclusão: \\
Conclui-se que escolares que necessitam de óculos apresentam maior \\
probabilidade de ter doença ocular, sendo necessário um exame oftalmo- \\
lógico completo na infância realizado por oftalmologistas capacitados \\
para a detecção e tratamento das diversas afecções encontradas além da \\
prescrição adequada dos óculos.
\end{tabular}

Descritores: Saúde escolar; Promoção da saúde; Óculos; Oftalmopatias/reabilitação; Acuidade visual

\section{INTRODUÇ̃̃̃O}

Entre 7 e $22 \%$ das crianças em idade escolar apresentam algum tipo de distúrbio visual. Estudos mostram que dentro de 100 escolares, por volta de 5 a 10 deles necessitam de correção visual por conta de erros refracionais (hipermetropia, miopia, astigmatismo) ${ }^{(1-9)}$. Destes, $5 \%$ apresentam baixa de acuidade visual de $50 \%$ ou mais, secundária a diversas afecções oculares ${ }^{(1-2)}$ 
A visão é responsável por $80 \%$ do contato do homem com o mundo exterior, portanto, afecções da mesma virão a comprometer o aprendizado e as atividades sociais. Estima-se que $10 \%$ das crianças na idade escolar apresentam alguma perturbação oftalmológica (erros de refração, estrabismo, ambliopia, seqüela de acidente ocular, malformações congênitas, entre outros). Os problemas visuais podem trazer sérias conseqüências para o desenvolvimento escolar bem como da auto-estima dos afetados, atingindo a qualidade de vida dos mesmos assim como a de seus familiares ${ }^{(10-13)}$.

Dados da OMS mostram que as pessoas com baixa acuidade visual têm em média um menor nível socioeconômico-cultural que aquelas que apresentam acuidade visual normal(14-15).

A assistência primária ocular permite a detecção de problemas oftalmológicos que adequadamente manejados virão a contribuir para o desenvolvimento socioeducacional normal dos escolares afetados ${ }^{(10-13)}$.

Temporini considera que os programas de Saúde Pública em oftalmologia devem priorizar ações relacionadas à prevenção de estados conducentes à cegueira e à incapacidade visual, a promoção de saúde ocular, a organização de assistência oftalmológica e a reabilitação de deficientes visuais ${ }^{(16)}$.

No Brasil, o sistema de Saúde Pública não contempla o atendimento oftalmológico aos escolares como programa estruturado, sendo o surgimento das "Campanhas" um ato de cidadania, a fim de detectar precocemente afecções oculares nos mesmos e fornecer tratamento adequado ${ }^{(3)}$.

No presente estudo, foram selecionadas crianças atendidas na "Campanha Olho no Olho" realizada em Recife no ano de 2004 .

\section{OBJETIVOS}

\section{Objetivo geral}

Verificar associação entre a necessidade de óculos e presença de outras afecções oculares encontradas em crianças atendidas na Campanha Olho no Olho realizada em Recife no ano de 2004.

\section{Objetivos especificos}

Determinar a freqüência da indicação de óculos entre escolares atendidos na Campanha Olho no Olho realizada na cidade do Recife no ano de 2004.

Determinar a freqüência de afecções oftalmológicas outras além de erros refracionais encontrados entre escolares consultados na Campanha Olho no Olho realizada na cidade do Recife no ano de 2004.

\section{MÉTODOS}

Em 2003, existiam 12.695 escolares matriculados na $1^{\mathrm{g}}$ série do Ensino Fundamental de 171 escolas públicas na cidade do Recife. Foram triados pelos professores, previa- mente treinados, 11.487 escolares, sendo selecionadas para exame oftalmológico as crianças que apresentavam acuidade visual (corrigida ou não, medida pela tabela de Snellen) igual ou inferior a $0,7(20 / 30)$, em pelo menos um dos olhos, bem como crianças que apresentavam algum sintoma de astenopia, segundo apreciação do professor. Oftalmologistas foram responsáveis pelo atendimento de 2.438 escolares, realizado em 2004. Foi preenchido protocolo oftalmológico individual, do qual foram obtidos os dados em relação à necessidade de óculos e presença de outras doenças oculares.

Quanto à prescrição de óculos, foram seguidas as orientações do CBO (Conselho Brasileiro de Oftalmologia) e do Ministério da Educação, que orientaram prescrição de óculos para hipermetropia de 3,0 dioptrias ou mais, miopia de $-0,75$ dioptrias ou mais e astigmatismos de $-0,75$ dioptrias em diante, com exame sob cicloplegia ${ }^{(13,17)}$. Para cicloplegia e midríase foi utilizado colírio de ciclopentolato, uma gota a cada 10 minutos por duas vezes, e uma gota de tropicamida, sendo realizado o exame em média uma hora após a segunda gota de ciclopentolato.

Foi considerada doença oftalmológica qualquer alteração da estrutura ou função normal do sistema visual diferentes de erros de refração, com exceção da anisometropia e da alta miopia. Foi considerado anisometropia como a diferença de refração entre os dois olhos a partir de 2 dioptrias e a alta miopia como uma miopia acima de 6 dioptrias, podendo levar a lesões retinianas ${ }^{(18)}$. Essas foram consideradas como integrantes do grupo de doenças oftalmológicas. Foi realizado exame de motilidade ocular, biomicroscopia com lâmpada de fenda marca Zeiss ${ }^{\circledR}$, oftalmoscopia binocular indireta com oftalmoscópio binocular Heine ${ }^{\circledR}$ e lente de 20 dioptrias marca $\operatorname{Volk}^{\circledR}$. Tonometria de aplanação somente foi realizada em casos isolados, com indicação da mesma.

Foram excluídas as fichas cujos dados não estiveram corretamente preenchidos, passando a constituir a amostra do estudo 1.994 crianças.

Os pacientes foram divididos em dois grupos, a saber: grupo $1 \mathrm{com}$ necessidade de óculos e grupo $2 \mathrm{sem}$ necessidade de óculos, relacionados com presença ou ausência de doença ocular.

Trata-se de um estudo descritivo de delineamento transversal que teve seus resultados analisados a partir do programa estatístico Epi Info versão 6.04, 1997.

\section{RESULTADOS}

Das 171 escolas públicas participantes com 12.695 alunos matriculados, foram triadas 11.478 crianças, das quais 2.438 foram consultadas na Campanha Olho no Olho em Recife realizada no ano de 2004, sendo que 1.994 alunos preenchiam os critérios de inclusão para o estudo.

Das 1.994 crianças deste estudo, houve necessidade de prescrição de óculos em 686 (34,40\% Grupo 1), sendo que $543(27,20 \%)$ não apresentaram qualquer outra afecção ocu- 
lar associada, enquanto $143(7,17 \%)$ tinha alguma doença ocular. Em 1.308 crianças $(65,50 \%$ Grupo 2$)$ não houve necessidade de óculos. Destas, $1.256(62,50 \%)$ não mostraram doença oftalmológica, enquanto $52(2,60 \%)$ apresentavam algum tipo de afecção ocular.

Foi encontrada uma necessidade de prescrição de óculos em $686(34,40 \%)$ dos 1.994 escolares participantes do estudo. Considerando todos os alunos (11.487) o percentual de óculos necessário foi de 5,97\%.

Foi calculado o Risco Relativo (RR) entre os grupos obtendo-se como resultado 5,24 (Intervalo de Confiança de 95\%: 3,87 a 7,10), sendo estatisticamente significativo $(p=0,0000001)$. Estes dados podem ser observados na tabela 1.

As doenças oculares encontradas nos pacientes mostraram $4,50 \%$ (90 crianças) com ambliopia, 3,20\% (65) com anisometropia, $2,00 \%$ (41) apresentaram estrabismo, $1,30 \%$ (26) com miopia degenerativa, $1,00 \%$ (20) com suspeita de glaucoma, $0,50 \%$ (10) com conjuntivite, $0,35 \%$ (7) com degeneração miópica do pólo posterior, $0,30 \%$ (6) com cicatriz de coriorretinite, $0,25 \%$ (5) com palidez papilar, $0,25 \%$ (5) com suspeita de ceratocone, $0,20 \%$ (4) com ptose palpebral e $0,20 \%$ (4) com leucoma corneano. Outras doenças encontradas como afacia, albinismo, membrana sub-retiniana, retinose pigmentar, catarata traumática, amaurose traumática, cicatriz macular, nistagmo, descolamento de retina e seclusão pupilar, apresentavam percentual menor que $0,15 \%$ (3). Esses dados se encontram na figura 1.

\section{DISCUSSÃO}

Foi verificado que a necessidade de óculos e a presença de outra doença ocular apresentou uma forte associação, expressa por um risco relativo de 5,24, mostrando que uma criança que necessita de óculos possui 5,24 vezes mais probabilidade de apresentar algum tipo de doença ocular quando comparada com uma criança sem necessidade dos mesmos, sendo este dado estatisticamente significativo (Intervalo de Confiança de $95 \%=3,87$ a 7,$10 ; p=0.0000001$ ). A associação encontrada destaca a importância de uma criança escolar receber atendimento de um profissional capacitado para detectar e tratar essas afecções oculares.

A prescrição de óculos teve um porcentual de 5,97\%, o que se aproxima dos dados de Campanhas semelhantes realizadas em anos anteriores no estado de Pernambuco. Em 1999 e 2000, as taxas encontradas foram de $6,3 \%$ e $6,6 \%$ respectivamente.

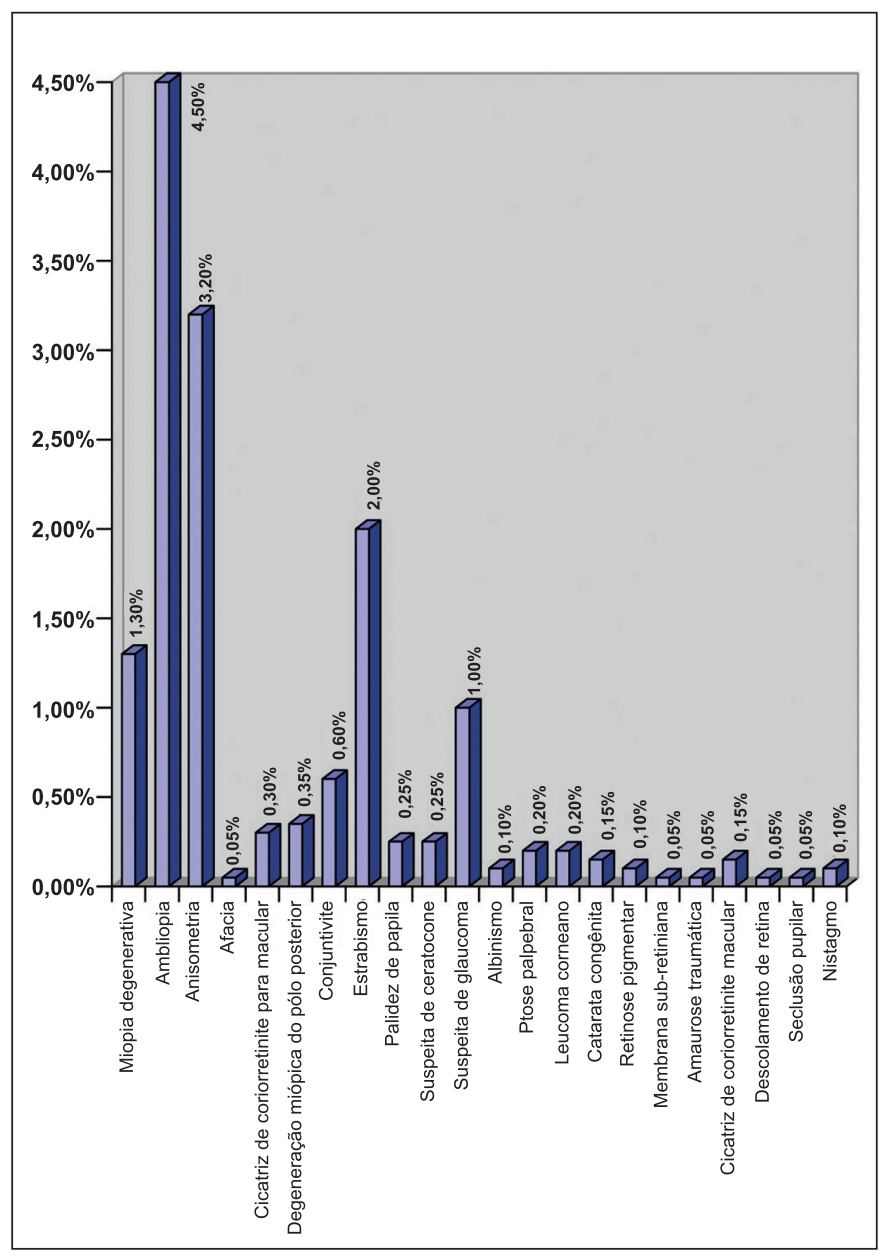

Figura 1 - Distribuição percentual das doenças oculares em 1.994 crianças consultadas na Campanha Olho no Olho em Recife no ano de 2004

Outros trabalhos da literatura também corroboram os resultados do presente estudo ${ }^{(15,19)}$.

A presença de doenças oculares em 195 (9,77\%) crianças reforça a importância do exame oftalmológico na infância. Mostra-se assim que a necessidade de óculos está intimamente relacionada com presença de doenças oculares e acredita-se que muitas vezes apenas o exame refratométrico pode ser insuficiente para a detecção dessas doenças, sendo, portanto, do ponto de vista de saúde pública, imprescindível o exame oftalmológico completo nas crianças.

Tabela 1 - Associação entre a necessidade de óculos e doença ocular em escolares atendidos na Campanha Olho no Olho em Recife no ano de 2004

\begin{tabular}{|c|c|c|c|c|c|c|}
\hline \multirow{2}{*}{$\begin{array}{l}\text { Necessidade de } \\
\text { óculos }\end{array}$} & \multicolumn{4}{|c|}{ Doença ocular } & \multirow[b]{2}{*}{$\mathbf{R R}$} & \multirow{2}{*}{$\begin{array}{r}\text { IC de } \\
95 \%\end{array}$} \\
\hline & \multicolumn{2}{|c|}{ Sim } & \multicolumn{2}{|c|}{ Não } & & \\
\hline Positivo (Grupo 1) & 143 & 7,17 & 543 & 27,20 & 5,24 & $3,87-7,10$ \\
\hline
\end{tabular}


Entre as diversas afecções oculares encontradas merece destaque a ambliopia com $4,5 \%$ e o estrabismo com $2,0 \%$. A importância das mesmas deve-se ao fato de não somente apresentarem probabilidade de levar ao déficit visual permanente, mas também com importante chance de apresentar resposta satisfatória se submetidas ao tratamento adequado precocemente. Esse dado é menor que o relatado em estudo realizado em Juiz de Fora, no qual mostra incidência de ambliopia de $6,8 \%$ em 1.875 crianças estudadas $^{(20)}$.

\section{CONCLUSÃO}

Conclui-se que escolares que apresentam necessidade de óculos apresentam maior probabilidade de outra doença oftalmológica associada mostrando que o exame oftalmológico completo na infância deve ser realizado por oftalmologistas capacitados para a detecção e tratamento das diversas doenças encontradas além da prescrição adequada dos óculos.

\section{ABSTRACT}

Purposes: To verify the relation between prescription of eyeglasses and presence of ocular infections found in the "Eye to Eye Campaign" carried out in the city of Recife in 2004. Methods: School-aged children seen in the "Eye to Eye Campaign" in Recife, in 2004. Stages recommended by the Brazilian Ophthalmologic Council (CBO) were followed, from preparation of teachers to select children to appointment with ophthalmologists. The patients were divided into two groups according to the need of eyeglasses, and related to presence or not of ocular conditions. This is a cross-section study and the results were analyzed by the statistical software Epi Info version 6.0. Results: In a group of 1994 school-aged patients, 686 needed eyeglasses (34.4\% - Group 1), 543 (27.2\%) did not had any ocular diseases, while $143(7.17 \%)$ had some ocular condition. A total of 1308 children (65.5\% - Group 2) did not require eyeglasses. Among those, 256 (62.5\%) did not show any ophthalmologic condition, whereas $52(2.6 \%)$ had some type of ocular diseases. Groups 1 and 2 were compared and children in need of eyeglasses had a 5.24-fold $(95 \%$ confidence interval: 3.87 to 7.10 ) greater relative risk of developing ocular diseases than children who did not require correction, and the difference was statistically significant $(\mathrm{p}=0.0000001)$. Conclusion: We conclude that school-aged children in need of eyeglasses are more likely to suffer ocular diseases, and a complete ophthalmologic examination should be performed in childhood by qualified ophthalmologists to detect and treat several disorders and also prescribe adequate correction.

Keywords: School health; Health promotion; Eyeglasses; Eye diseases/rehabilitation; Visual acuity.

\section{REFERÊNCIAS}

1. Neurauter R, Bellini AT, Couto-Júnior AS. Saúde ocular de pré-escolares e escolares dos bairros de Copacabana e Urca - Rio de Janeiro. Rev Bras Oftalmol. 2001;60(3):199-203.

2. Alberto FL, Callera F, Daré GMR, Rodrigues MLV. Estudo das condições oculares em uma população de estudantes do $1^{\circ}$ grau na cidade de Ribeirão Preto - SP. Arq Bras Oftalmol. 1992;55(4).

3. Alves MR, Kara-José N. Manual de Instruções. Conselho Brasileiro de Oftalmologia. Campanha Nacional de Prevenção de Cegueira e Reabilitação Visual, 1998.

4. Couto Júnior AS. Considerações preliminares sobre a identificação das deficiências visuais em pré-escolares e escolares [monografia]. Rio de Janeiro: Instituto Benjamin Constant, 1992.

5. Rocha H, Ribeiro-Gonçalves E, coordenadores. Ensaio sobre a problemática da cegueira: prevenção, recuperação, reabilitação. Belo Horizonte: Fundação Hilton Rocha; 1987.

6. Rossi AG, et al. Avaliação oftalmológica em 1.000 crianças escolares da rede municipal de Santa Maria - RS. Arq Bras Oftalmol. 1996;59(4). [XII Congresso Brasileiro da Cegueira e I Congresso Panamericano de Prevenção da Cegueira realizado em setembro de 1996].

7. Suzuki CK, et al. Saúde ocular de alunos da primeira a oitava séries do primeiro grau de escolas estaduais de São Paulo - SP. Arq Bras Oftalmol. 1996;59(4). [XII Congresso Brasileiro da Cegueira e I Congresso Panamericano de Prevenção da Cegueira realizado em setembro de 1996].

8. Suzuki CK, Santos Neto E. Estudo da acuidade visual de escolares da rede estadual e da rede particular de ensino do município de Barueri - SP, 1991. Rev Bras Saúde Esc. 1994;3(1/4):244-51.

9. Vieira C, Rodrigues MLV. Prevenção da cegueira nas escolas rurais da região de Santa Bárbara D'oeste - SP. Rev Bras Oftalmol. 1995;54(2):43-7.

10. Alves MR, Kara-José N. O olho e a visão: o que fazer pela saúde ocular de nossas crianças. Petrópolis: Vozes; 1996.

11. Kara-José N, et al. Manual da boa visão. Campinas: Editora Unicamp; 1991.

12. Kara-José N, Oliveira RSC. Mitos e verdades em oftalmologia. São Paulo: Contexto; 1997.

13. Brasil. Ministério da Saúde. Secretaria de Assistência à Saúde. Departamento de Assistência e Promoção à Saúde. Coordenação de Doenças Crônico-Degenerativas. Informações básicas para a promoção da saúde ocular. Secretaria de Estado de Saúde de São Paulo. Serviço de Oftalmologia Sanitária. Brasília: Coordenação de Documentação e Informação do Ministério da Saúde; 1994.

14. Burns MJ. Building a priority for a national vision health care. Eye Ear Nose Throat Mon. 1973;52(10):353-6.

15. Beer SMC. Saúde ocular de crianças entre zero e seis anos de idade, residentes na cidade de São Caetano do Sul, SP, Brasil [tese]. São Paulo: Universidade Federal de São Paulo. Escola Paulista de Medicina; 2000.

16. Temporini ER. Pesquisa de oftalmologia em Saúde Pública: considerações metodológicas sobre fatores humanos. Arq Bras Oftalmol. 1991;54(6):279-81.

17. Alves MR, Kara-José N. Campanha "Veja bem Brasil". Manual de Orientação. São Paulo: Conselho Brasileiro de Oftalmologia; 1998.

18. Alves AA. Refração. Rio de Janeiro: Cultura Médica; 1994. p.70, 83. [Série Biblioteca Brasileira de Oftalmologia].

19. Nassaralla Júnior JJ, Nasssaralla BRA. Atendimento oftalmológico no sistema público de ensino em Goiânia - Projeto Boa Visão. Rev Bras Oftalmol. 2002;61(6):446-52.

20. Alves MHF. Incidência de ambliopia em crianças examinadas no Serviço de Oftalmologia Social de Juiz de Fora. Rev Bras Oftalmol. 1996;55(10):775-80. 